\title{
Observability and Estimation Methods Using Synchrophasor *
}

\author{
Kai Sun* Wei Kang** \\ * Department of EECS, The University of Tennessee, Knoxville, TN \\ 37996 USA (e-mail: kaisun@utk.edu). \\ ** Department of Applied Mathematics, Naval Postgraduate School, \\ Monterey, CA 93943 USA (e-mail: wkang@nps.edu)
}

\begin{abstract}
Intermittent generations such as large-scale renewable energy increases the risk of instability in a power grid. In this paper, we introduce the concept of observability and its computational algorithms for power networks equipped with synchrophasors. The goal is to estimate the angles and its derivatives around unstable trajectories, the information that is critical for the detection of power network instabilities. The algorithm is developed to determine the number and the siting of synchrophasors in a power network so that the state of the system can be accurately estimated in the presence of instability. An unscented Kalman filter (UKF) is adopted as a tool to estimate the states that are not directly measured by synchrophasors. The theory and its computational algorithms are illustrated by using a 9-bus model with three generators.
\end{abstract}

\section{INTRODUCTION}

The penetration of a large number of intermittent power generations using renewable energy or green technologies brings increasing uncertainties to daily operations of interconnected power transmission systems. Early indicating wide-area stability problems (e.g. cascading events) is crucially important for control centers to prevent power outages and blackouts. In the US and many other countries, synchrophasors are being increasingly installed in transmission systems to provide wide-area real-time measurement data synchronized by GPS. In the next five to ten years, thousands of synchrophasors will be installed in North America. Electricity utilities are building synchrophasor networks to collect data from dispersed synchrophasors and then send the huge volume of data to online stability applications at control centers. Monitoring, analysis and control are three key operational functions of control centers for the detection, prediction and attenuation of stability problems. As of today there are still large technical gaps in applying synchrophasor data for online analysis and real-time network control. Most current synchrophasor applications are based on direct visualization for online monitoring, e.g. displaying angle differences between selected power plants/substations and drawing voltage/frequency contours. These methods cannot provide the information about the dynamic behavior of a power network with intermittent generations. On the other hand, the data in time series collected from synchrophasors makes it possible to reliably predict the dynamic behavior of networks. The goal of this paper is to develop and to validate the theory and the methodology of designing efficient synchrophasor networks and data fusion algorithms.

\footnotetext{
* This work was supported in part by EPRI Program on Technology Innovation 3002002061. The second author participated this project as an independent consultant.
}

How to evaluate networked synchrophasors in a largescale grid is a fundamental question in this study. More specifically, the following issues present challenges and opportunities that motivate the research topics in this paper:

- What is the minimum requirement in terms of size and siting for a synchrophasor network to guarantee that variables at system instability are credibly observable?

- How to optimally design a synchrophasor network based on a given number of synchrophasors to achieve the maximum observability at instability?

- Develop computationally efficient algorithms for the estimation of state variables that are not directly measured by sensors.

While the theory and algorithms for the filtering and optimal sensor design have been developed for many years, some recent results in Kang-Xu [2009a,b] can be used to quantitatively measure observability for large-scale nonlinear systems such as numerical weather prediction Kang-Xu [2012] and power grids. In this paper, we extend these results to explore and analyze the observability of power networks and to address some of the afore mentioned issues on synchrophasor network design.

In this paper, we first introduce the concept of observability and its computational algorithm. This concept is fundamental to the evaluation of synchrophasor networks. It is used to determine the number and the siting of synchrophasors in a network so that the state of the system can be accurately estimated in the presence of instability. Then, an unscented Kalman filter (UKF) is introduced as a tool to estimate the states that are not directly measured by synchrophasors. We demonstrate the methodology developed in this paper using a 9-bus model with three generators. 


\section{OBSERVABILITY AND UKF}

In this section, we introduce the concept of unobservability index and a state estimation method based on an unscented Kalman Filter.

\subsection{Observability}

In Kang-Xu [2009a,b], a quantitative measure of partial observability is defined for general dynamical systems. For power systems, we adopt a simplified version of that definition. Consider any system defined by ordinary differential equations

$$
\begin{aligned}
& \frac{d x(t)}{d t}=f(t, x(t)), \\
& y(t)=h(x(t))
\end{aligned}
$$

where $x \in \mathbb{R}^{n}$ is the state variable, $y(t) \in \mathbb{R}^{m}$ is the system's output given by sensor measurement. In this section, we define the observability of initial conditions, $x(0)$, which uniquely determine the trajectories of a system. The definition can be easily modified to define the observability of $x\left(t_{0}\right)$ for any given time $t=t_{0}$. Suppose $x$ and $y$ lie in normed spaces with norms denoted by $\|x\|$ and $\|y\|_{Y}$.

Definition 1. Given $\rho>0$ and a nominal trajectory $x(t)$ of (1). Let

where $\hat{x}(t)$ satisfies

$$
\epsilon^{N}=\inf \|h(\hat{x}(t))-h(x(t))\|_{Y}
$$

$$
\begin{aligned}
& \frac{d \hat{x}}{d t}=f(t, \hat{x}) \\
& \|\hat{x}(0)-x(0)\|=\rho
\end{aligned}
$$

Then $\rho / \epsilon$ is called the unobservability index of $x(0)$.

Remark. This is a quantitative measure of observability. The ratio $\rho / \epsilon$ can be interpreted as follows: if the maximum error of the measured output, or sensor error, is $\epsilon$, then the worst estimation error of $x(0)$ is $\rho$. Therefore, a small value of $\rho / \epsilon$ implies strong observability of $x(0)$. For linear systems with a $L^{2}$-norm, it can be proved that the reciprocal of the unobservability index is the square root of the smallest eigenvalue of the observability gramian.

Definition 1 can be numerically implemented for nonlinear systems. In this project, two algorithms are used to compute the observability, namely the empirical gramian method and the method of pseudospectral dynamical optimization. Empirical gramian is a method of first order approximation for the unobservability index. The idea is to approximate the unobservability index using the smallest eigenvalue of a gramian matrix Kang-Xu [2009a,b]. Due to its simplicity, this method is used for most simulations in this project. However, for the problem of robust observability the first order approximation is not accurate enough. As an alternative, we use a more sophisticated computational algorithm based on pseudospectral method Kang [2006].

\subsection{Unscented Kalman Filter}

For systems with strong or reasonable observability, filters can be used as virtual sensors to estimate the variables that are not directly measured. Kalman filter was developed originally for linear systems. Modified Kalman filters are widely used for nonlinear systems. Extended Kalman filters require the linearization of system models, which may not be easily available during real-time operations for large-scale systems like power grids. In this project, we adopt Unscented Kalman Filter (UKF) which does not require online computation of system linearization. Consider a nonlinear system

$$
\begin{aligned}
& x_{n}=f\left(x_{n-1}, w_{n-1}\right) \\
& y_{n}=h\left(x_{n-1}, v_{n-1}\right)
\end{aligned}
$$

where $x, y, w$ and $v$ are the state, measurement, process noise and measurement noise, respectively. The UKF is "founded on the intuition that it is easier to approximate a probability distribution than it is to approximate an arbitrary nonlinear function or transformation" JulierUhlmann [2004]. The algorithm of an UKF is outlined as follows. Details can be found in Julier-Uhlmann [2004].

- Based on the previous-step estimation of the state, $\hat{x}_{n-1}$, and the covariance matrix, $\hat{P}_{n-1}^{x x}$, calculate a set of sigma points as

$$
\sigma^{i}=\hat{x}_{n-1} \pm \sqrt{N_{x} \hat{P}_{n-1}^{x x}}, i=1,2, \ldots, N_{x} ;
$$

- Propagate all the sigma points through the nonlinear dynamic and the output equations,

$$
z^{i}=f\left(\sigma^{i}, 0\right), g^{i}=h\left(\sigma^{i}, 0\right), i=1,2, \ldots, N_{x}
$$

- Calculate the mean (prediction) of the state and output,

$$
\tilde{x}_{n}=\frac{1}{2 N_{x}} \sum_{i=1}^{2 N_{x}} z^{i} \quad \tilde{y}_{n}=\frac{1}{2 N_{x}} \sum_{i=1}^{2 N_{x}} g^{i} ;
$$

- The prediction of the covariance matrices are given by,

$$
\begin{aligned}
& \tilde{P}_{n}^{x x}=\frac{1}{2 N_{x}} \sum_{i=1}^{2 N_{x}}\left(z^{i}-\tilde{x}_{n}\right)\left(z^{i}-\tilde{x}_{n}\right)^{T} \\
& \tilde{P}_{n}^{y y}=\frac{1}{2 N_{x}} \sum_{i=1}^{2 N_{x}}\left(g^{i}-\tilde{y}_{n}\right)\left(g^{i}-\tilde{y}_{n}\right)^{T} \\
& \tilde{P}_{n}^{x y}=\frac{1}{2 N_{x}} \sum_{i=1}^{2 N_{x}}\left(z^{i}-\tilde{x}_{n}\right)\left(g^{i}-\tilde{y}_{n}\right)^{T}
\end{aligned}
$$

Once the prediction of $\tilde{x}_{n}, \tilde{P}_{n}^{x x}, \tilde{P}_{n}^{y y}$ and $\tilde{P}_{n}^{x y}$ are available, the update is given by

$$
\hat{x}_{n}=\tilde{x}_{n}+K\left(y_{n}-\tilde{y}_{n}\right)
$$

where

$$
K=\tilde{P}_{n}^{x y}\left[\tilde{P}_{n}^{y y}\right]^{-1}, \hat{P}_{n}^{x x}=\tilde{P}_{n}^{x x}-K \tilde{P}_{n}^{x y} K^{T} .
$$

In this paper, UKF is used as virtual sensors to estimate both state variables and uncertain parameters. It is also used as a tool to verify the observability computed using different methods.

\section{OBSERVABILITY ANALYSIS ON THE BOUNDARY OF THE DOMAIN OF ATTRACTION}

To provide adequate information for stability analysis and control, it is important to verify that the system is reasonably observable at the time of instability. Given a set of synchrophasors, the unobservability index can be computed to determine if the system is adequately 
observable. This approach is tested and illustrated using the following system of a 9-bus model.

\subsection{A 9-bus model}

The model is adopted from Anderson-Fouad [1994]. The dynamical system is defined using a set of ODEs

$$
\begin{aligned}
& \frac{2 H_{i}}{\omega_{R}} \frac{d \omega_{i}}{d t}+D_{i} \omega_{i}=P_{m i}-P_{e i}, \\
& \frac{d \delta_{i}}{d t}=\omega_{i}-\omega_{R}, \quad i=1,2,3
\end{aligned}
$$

where

$$
\begin{gathered}
P_{e i}=E_{i}^{2} G_{i i}+\sum_{j=1, j \neq i}^{n} E_{i} E_{j} Y_{i j} \cos \left(\theta_{i j}-\delta_{i}+\delta_{j}\right) \\
D_{i}=0, i=1,2,3 ; \\
H_{1}=47.28, H_{2}=12.8, H_{3}=6.02 \\
\omega_{R}=60 \cdot(2 \pi) \\
E_{1}=1.0566, E_{2}=1.0502, E_{3}=1.0170 \\
\text { Base }=100 \mathrm{MVA} \\
P_{1}=\frac{71.6}{\text { Base }}, P_{2}=\frac{163.0}{\text { Base }}, P_{3}=\frac{85.0}{\text { Base }}
\end{gathered}
$$

The reduced $Y$ matrix is

$$
Y=\left[\begin{array}{lll}
0.8455-2.9883 J & 0.2871+1.5129 J & 0.2096+1.2256 J \\
0.2871+1.5129 J & 0.4200-2.7239 J & 0.2133+1.0879 J \\
0.2096+1.2256 J & 0.2133+1.0879 J & 0.2770-2.3681 J
\end{array}\right]
$$

The system has an equilibrium

$$
\begin{aligned}
& {\left[\begin{array}{lll}
\delta_{1} & \delta_{2} & \delta_{3}
\end{array}\right]^{T}=\left[\begin{array}{lll}
2.2717^{\circ} & 19.7315^{\circ} & 13.1752^{\circ}
\end{array}\right]^{T}} \\
& \omega_{i}=\omega_{R}, \quad i=1,2,3
\end{aligned}
$$

\subsection{The domain of attraction}

Around any equilibrium of nonlinear systems there is a domain of attraction. The trajectory starting from any point in the domain converges to the equilibrium. However, there is no general way of computing the domain of attraction. Although Zubov's equation defines the boundary of the domain, this equation is extremely difficult to solve, numerically or analytically, if not impossible. On the other hand, practical criterions based on experimentations can be used to approximate a domain of attraction. It is desired that the data collected by synchrophasors make the system strongly observable at the boundary of the domain of attraction, which implies that all state information can be reliably estimated for analysis and control before a trajectory becomes unstable.

In the following, we find a layer outside the boundary of the domain of attraction. Starting from this layer, all trajectories lost its stability. After tens of thousands of simulations, what we found for the 9-bus system is that stability is lost quickly after at least one relative angle becomes larger than $650^{\circ}$. Therefore, we use the following practical criterion to compute an envelop outside the domain of attraction: all initial angles so that at least one relative angle is between $650^{\circ}$ and $750^{\circ}$ at $t=5 \mathrm{~s}$. In the computation, if we choose a time interval longer than $t=5$, the envelope is tighter, i.e. it is closer to the boundary of the domain of attraction. For a time interval shorter than $t=5$, the envelope becomes loose. We found that $t=5$ is a good time interval which is long enough to provide adequate information for analysis and, meanwhile, it is short enough to achieve fast throughput for computations.

In this paper, we focus on the domain of attraction in $\delta_{1} \delta_{2} \delta_{3}$-space with a fixed $\omega$. In this case, the equilibrium is

$$
\delta_{e}=\left[\begin{array}{lll}
2.2717^{\circ} & 19.7315^{\circ} 13.1752^{\circ}
\end{array}\right]^{T}
$$

The domain of attraction in the $\omega-\delta$ space has higher dimension, which is addressed in Kang-Sun [2013]. It is omitted due to space limitation. In the computation, the boundary of the domain of attraction is approximated by finite many points around the equilibrium (6). The grid points for $\delta_{3}$ is a sequence in the interval $\left[-92.2462^{\circ} 136.9369^{\circ}\right]$, i.e.

$$
\delta_{3}^{k}=-92.2462^{\circ}+14.3239^{\circ} k, \quad k=0,1,2, \cdots, 16
$$

For each value of $\delta_{3}$, a $360^{\circ}$ search around the equilibrium (6) is carried out numerically for a sequence of directions,

$$
\begin{aligned}
& v_{j}=\left[\cos \left(\theta_{j}\right) \sin \left(\theta_{j}\right) 0\right], j=1,2, \cdots, 120 \\
& \theta_{j}=3 j,
\end{aligned}
$$

For each $\delta_{3}^{k}$ in (7), one point in the envelope of the domain of attraction is found in the direction of $v_{j}$,

$$
\left[\begin{array}{lll}
2.2717^{\circ} & 19.7315^{\circ} \delta_{3}^{k}
\end{array}\right]+r v_{j}
$$

in which the radius $r$ is determined as follows.

(1) Find $r_{\min }$ and $r_{\max }$ so that the system is stable with the initial condition (8) using $r_{\min }$; and unstable using $r_{\max }$.

(2) Let $r=\frac{1}{2}\left(r_{\max }+r_{\min }\right)$. Solve ODE (3) using initial condition (8).

(3) Check $\Delta=\max \left\{\left|\delta_{2}(T)-\delta_{1}(T)\right|,\left|\delta_{3}(T)-\delta_{1}(T)\right|\right\}$. We set $T=5$ seconds.

- If $\Delta>750^{\circ}$, then $r_{\max }=r$, go to step 2 .

- If $\Delta<650^{\circ}$, then $r_{\min }=r$, go to step 2 .

- If $650^{\circ} \leq \Delta \leq 750^{\circ}$, stop. The point (8) is in the envelope of the domain of attraction.

This algorithm is applied to 16 grid points of $\delta_{3}^{k}$ with 120 different directions $v_{j}$, a total of $17 \times 120=2040$ combinations. The envelope of the domain of attraction is shown in Figure 2. Trajectories starting from this surface diverge after about $t=5$ seconds. A typical trajectory is shown in Figure 1.

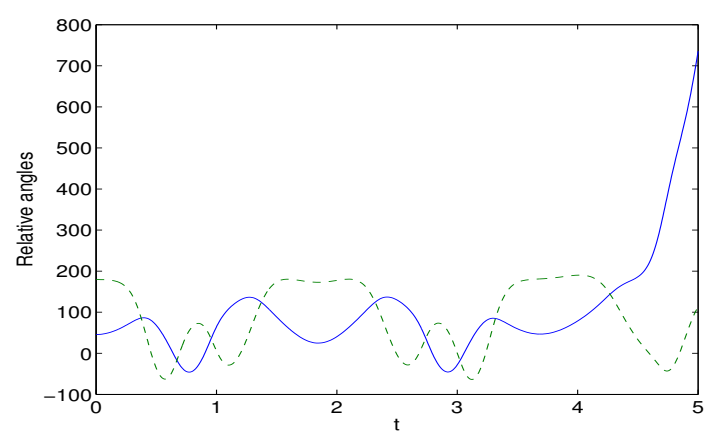

Fig. 1. Relative angles of an unstable trajectory. Solid line: $\delta_{2}-\delta_{1} ;$ dashed line: $\delta_{3}-\delta_{1}$ 


\subsection{Observability}

For the purpose of detecting instability, the synchrophasors should be installed so that the unstable trajectories close to the domain of attraction should be observable. In the following, the quantitative observability in Definition 1 is applied to all points in the envelope of the domain of attraction. The unobservability index is numerically computed using the empirical gramian method Kang-Xu [2009a,b]. Let us assume that a synchrophasor is installed at Generator 1, i.e. $\delta_{1}$ and $\omega_{1}$ can be measured by sensors. In this case the output function is

$$
y=\left[\begin{array}{ll}
\omega_{1} & \delta_{1}
\end{array}\right]^{T}
$$

We assume that the sensor collects data at a rate of $30 \mathrm{~Hz}$. The norms in Definition 1 are defined as follows.

$$
\|y(t)\|_{Y}^{2}=\frac{1}{30} \sum_{j=1}^{30}\left[\begin{array}{ll}
\omega_{1} & \delta_{1}
\end{array}\right] W_{1}^{2}\left[\begin{array}{c}
\omega_{1} \\
\delta_{1}
\end{array}\right]
$$

where $W_{1}$ is the weight matrix

$$
W_{1}=\left[\begin{array}{cc}
1 / R_{\omega} & 0 \\
0 & 1 / R_{\delta}
\end{array}\right], \quad \begin{aligned}
& R_{\omega}=2 \pi \cdot 5 \cdot 10^{-3} \\
& R_{\delta}=0.01 \cdot \frac{\pi}{180}
\end{aligned}
$$

This weight matrix is chosen based on the assumption that the sensor error for $\omega$ is bounded by $5 \times 10^{-3} \mathrm{~Hz}$ and the error of $\delta$ is bounded by $0.01^{\circ}$. The metric for state variables is

$$
\|x\|^{2}=x^{T} W_{2}^{2} x
$$

where $x=\left[\begin{array}{llllll}\omega_{1} & \omega_{2} & \omega_{3} & \delta_{1} & \delta_{2} & \delta_{3}\end{array}\right]^{T}$ and the weight matrix, $W_{2}$, is defined as follows,

$$
W_{2}=\left[\begin{array}{cc}
\frac{50}{R_{\omega}} I_{3} & 0 \\
0 & \frac{50}{R_{\delta}} I_{3}
\end{array}\right], I_{3} \text { is an identity matrix }
$$

This weight matrix implies that $50 \times 0.01=0.5^{\circ}$ and $50 \times 5 \times 10^{-3}=0.25 \mathrm{~Hz}$ are considered good accuracy in estimation. The unobservability index, $\rho / \epsilon$, in Definition 1 is a number describing the smallest input-to-output gain from the initial state to the variables measured by sensors. It means that the worst estimation error of the state variable is $\rho / \epsilon$ times the sensor error, in their corresponding metrics. For the purpose of instability analysis, the goal is to have reasonable estimate that can tell the trends of the state variables. For this purpose, trajectories with $\rho / \epsilon \in[0,1]$ are strongly observable; $1 \leq \rho / \epsilon \leq 30$ are reasonably observable; $\rho / \epsilon>30$ are weakly observable, sometimes unobservable.

The envelope of the domain of attraction computed in Section 3.2 consists of the initial states from which the trajectories lost stability at $t=5$. As shown in Figure 1, the trends of these trajectories in the time interval $[4,5]$ is important. Therefore, we compute the unobservability index for this time interview. The result is shown in Figure 2 . The value of unobservability index for each point in the envelope is represented by different colors, cold color representing strongly observable and warm color representing weakly observable. The range of the unobservability index is between 0.49 and 22.66. Therefore, all unstable trajectories are reasonably observable. In fact, from the figure one can see that all points in the envelope are strongly observable except for a few spots. More specifically, Figure 3 is the histogram of the unobservability index. It shows that about 900 points in the envelope have unobservability index close to $\rho / \epsilon=4$, which is a good observability. Very few points have an index larger than twenty.

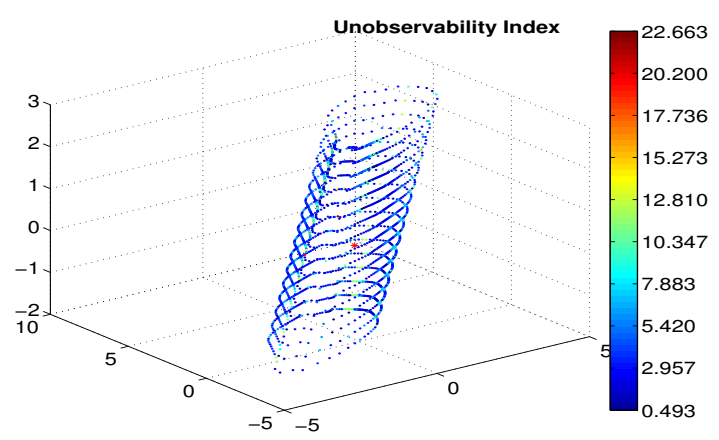

Fig. 2. An envelope of the domain of attraction in $\delta_{1} \delta_{2} \delta_{3^{-}}$ space and its unobservability indices

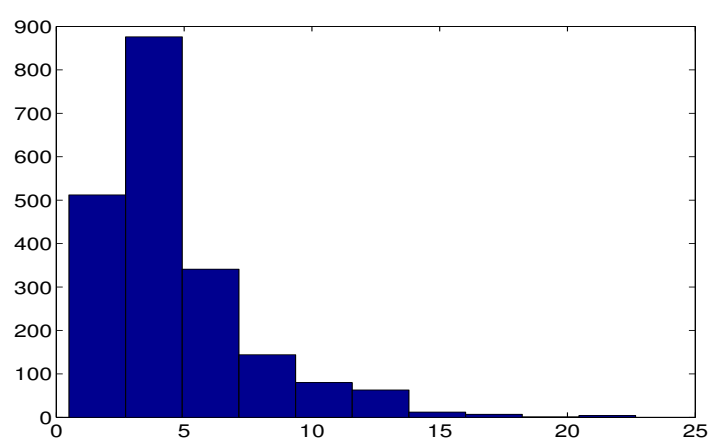

Fig. 3. The histogram of unobservability index

If the synchrophasor is installed at Generator 2 or 3 , the unstable trajectories are also observable. The results are shown in Figure 4 and 5 . In fact, the observability in these two cases are better than the first case.

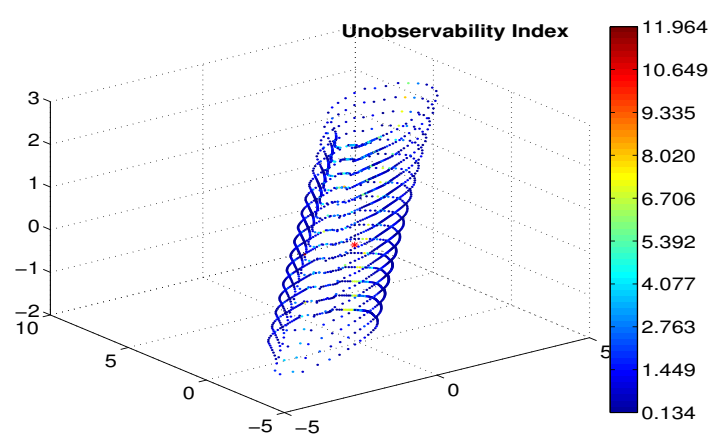

Fig. 4. Unobservability index of unstable initial conditions, sensor at the 2 nd generator

\section{OBSERVABILITY AND ESTIMATION IN THE PRESENCE OF SYSTEM UNCERTAINTIES}

System instabilities can be triggered by two different reasons, an initial state outside the domain of attraction or a change of system parameters. The instability caused by 


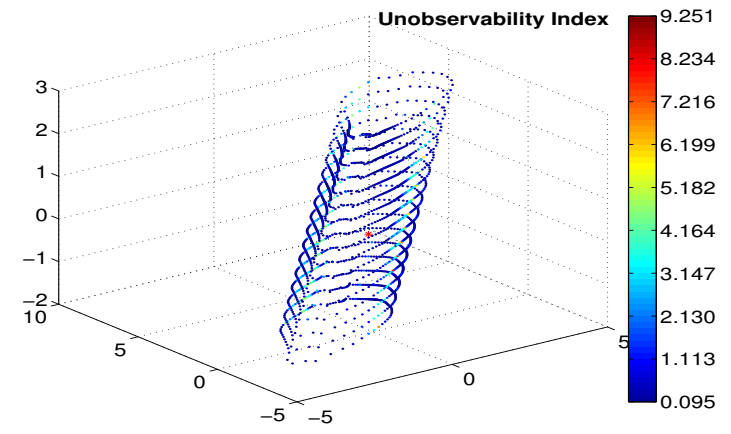

Fig. 5. Unobservability index of unstable initial conditions, sensor at the 3rd generator

initial states is studied in the previous section. Now we consider systems with unpredictable parameter changes. In the first subsection, we study the robustness of the observability in the presence of unknown parameter changes. In the second subsection, we introduce an adaptive nonlinear estimation method to provide accurate state estimate. In addition, some unknown parameters can also be estimated using sensor information.

\subsection{Robustness of observability}

Let $\delta_{i}^{e}$ and $\omega_{i}^{e}, i=1,2,3$, be the equilibrium point (5) of the system in (3) with a reduced $Y$ matrix (4). At time $t=0$, we assume that the system's admittance matrix is changed,

$$
\bar{Y}=Y+\Delta Y
$$

We assume that this change is not known to the operator. If the estimation is still based on the original $Y$ matrix, how robustness is the observability? To quantitatively measure the robustness of observability, we use the remainders of trajectories, an approach from Kang [2011]. Suppose the sensor measures $\delta_{1}(t)$ and $\omega_{1}(t)$. Let $\delta_{i}(t)$ and $\omega_{i}(t), i=1,2,3$, be a trajectory with the new $\bar{Y}$ matrix starting from the equilibrium (5). Suppose $\delta_{i}^{*}(t)$ and $\omega_{i}^{*}(t)$ be the best estimate of $\delta_{i}(t)$ and $\omega_{i}(t)$ using the original $Y$ matrix,

$$
\begin{aligned}
& \min \left\|\left[\tilde{\delta}_{1}(t)-\delta_{1}(t) \tilde{\omega}_{1}(t)-\omega_{1}(t)\right]\right\|_{W_{1}} \\
& \tilde{\omega}_{i}(t) \text { and } \tilde{\delta}_{i}(t) \text { satisfy }(3)-(4)
\end{aligned}
$$

where $W_{1}$ is defined in (10). Let $\omega_{i}^{r}(t)=\omega_{i}(t)-\omega_{i}^{*}(t)$ and $\delta_{i}^{r}(t)=\delta_{i}(t)-\delta_{i}^{*}(t)$ be the remainder, then

$$
\omega_{i}(t)=\omega_{i}^{*}(t)+\omega_{i}^{r}(t), \delta_{i}(t)=\delta_{i}^{*}(t)+\delta_{i}^{r}(t)
$$

According to $(13), \delta_{i}^{*}(t)$ and $\omega_{i}^{*}(t)$ are the best estimate of $\delta_{i}(t)$ and $\omega_{i}(t)$ using the matrix $Y$ and the sensor information. The estimation error is the remainder, $\delta_{i}^{r}(t)$ and $\omega_{i}^{r}(t)$. This error is not directly caused by the output noise, i.e. this error cannot be reduced no matter how accurate the output is measured. Therefore, the remainder is a measure of the robustness of observability. If the remainder is small, it implies that a nonlinear estimator is able to accurately estimate the state variables in the presence of small unknown parameter change.

To compute the remainder and the best estimate, we must solve (13). It is a problem of nonlinear dynamic optimization. An analytic solution does not exist. In this section, computational dynamic optimization is applied to solve (13). More specifically, we apply a pseudospectral method based on Legendre-Gauss-Lobatto quadrature nodes Fahroo-Ross [1998], Kang [2006]. As an example, we computed the remainder for a $\Delta Y$ in which all entries are zero except

$$
\Delta Y_{12}=\Delta Y_{21}=r Y_{12}, \quad r=-0.01
$$

i.e. the entries $Y_{12}$ and $Y_{21}$ in $Y$ are reduced by $1 \%$ in magnitude. For the time interval $[0,1]$, the remainder is shown in Figure 6. The magnitude of the remainders
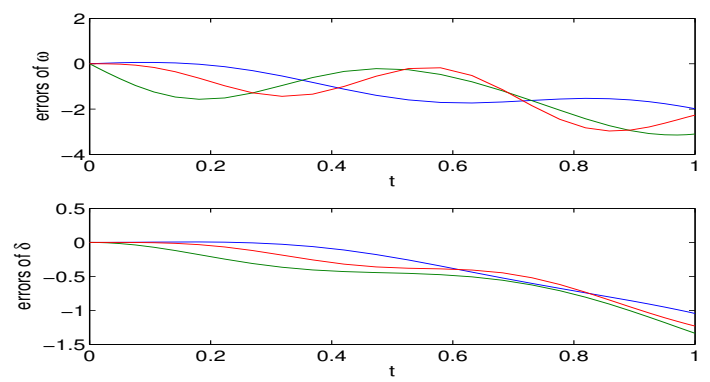

Fig. 6. The remainder for $t \in[0,1]$

depends on the length of the time interval. We found that it increases with the final time $T$. If we use the final-time error as a metric for the remainder, i.e.

$\left\|\left[\omega_{1}^{r}(T) \omega_{2}^{r}(T) \omega_{3}^{r}(T)\right]\right\|$ and $\left\|\left[\delta_{1}^{r}(T) \delta_{2}^{r}(T) \delta_{3}^{r}(T)\right]\right\|$ the results for $T=1,2,3,4,5$ is shown in Figure 7 . The unit in the figure is "deg" for $\delta$ and "deg/s" for $\omega$. In this figure, "o" represents the norm of angular velocities and "*" represents the norm of angles. The result implies that the unobservability index is "unstable" in the sense that the remainder's magnitude, i.e. the error of the best estimate, increase with time. Therefore, the observability decreases as time goes on. The angles increase linearly and the angular velocities increase at a higher order. Therefore, we conclude that the observability is not robust to the variation of system parameters.

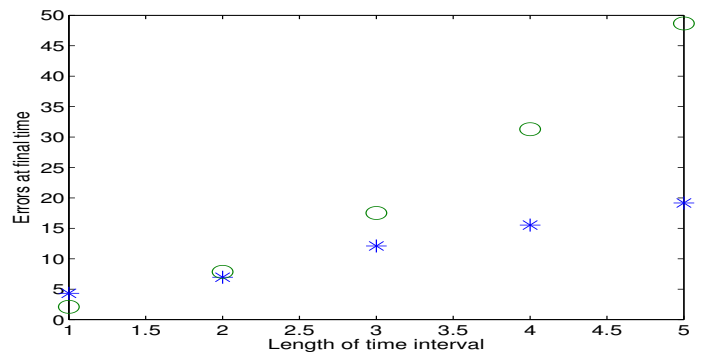

Fig. 7. The remainder for $T=1,2,3,4,5$

\subsection{The observability of unknown parameters and adaptive estimation}

Given the robustness study, it is important to make an estimator that is adaptive to the unknown parameter change. In fact, we can compute the observability of both the state variables and the parameters in the system model. More specifically, suppose the unknown parameter is the magnitude of $Y_{12}$, then

$$
\bar{Y}_{12}=r Y_{12}
$$

If $r=1$, the nominal value of $Y$ is the same as the true value. If $r \neq 1$, the parameter in the system is 


\begin{tabular}{|c|c|}
\hline Entry of $80 \%$ change & Unobservability Index \\
\hline$Y_{12}$ & 0.8572 \\
\hline$Y_{13}$ & 1.7593 \\
\hline$Y_{23}$ & 0.8055 \\
\hline
\end{tabular}

Table 1. Unobservability index of parameter variation and state variables

\begin{tabular}{|c|c|}
\hline Entries of $80 \%$ change & Unobservability Index \\
\hline$Y_{12}$ and $Y_{13}$ & 1.2585 \\
\hline$Y_{12}$ and $Y_{23}$ & 0.7026 \\
\hline$Y_{13}$ and $Y_{23}$ & 1.3826 \\
\hline
\end{tabular}

Table 2. Unobservability index of parameter variation and state variables

varied. In this case, we want to estimate the change and then adaptively adjust the estimates. For this purpose, we define the observability of both $\omega_{i}, \delta_{i}$, and $r$ using Definition 1 and the following norm

$$
\|x\|^{2}=x^{T} W_{2} x+W_{3} r^{2}
$$

$W_{2}$ is from (12) and $W_{3}=1 / 0.05$. The nominal value, $r_{e}$, in the simulations is 0.8 , i.e. we assume a $20 \%$ parameter variation that is unknown. Observability of the parameter variation as well as the state variables is computed and listed in Table 1. From the table we can conclude that parameter variations are observable. For instance, if $Y_{12}$ is varied by $80 \%$, the unobservability index is 0.9853 , which implies strong observability. If two parameters are unexpectedly changed by $80 \%$, the system is still observable. The result is shown in Table 2 .

\subsection{Adaptive estimation}

Because the unknown parameters are observable, it makes sense to apply an estimator that is adaptive to the system change. In this case the sensor information serves two purposes: for the estimation of the state variables and for the real-time update of parameter changes. Once again, UKF is applied to estimate the value of the state variable as well as the parameter variation.

We tested UKF by changing one parameter in $Y$ matrix by $20 \%$. For example, the true value of $\bar{Y}_{12}$ is $80 \%$ of $Y_{12}$ given to the UKF. Started from the incorrect model parameters, the UKF uses the sensor information with noise to estimate $\omega_{1}$ and $\delta_{i}$ as well as $Y_{12}$. The process is able to correct the parameter automatically so that the estimates gradually approach the true value. The result is shown in Figure 8. Similar simulations are carried out for the variation of all other parameters in $Y$. All estimates are convergent with a behavior similar to what shown in Figure 8 .

\section{CONCLUSION}

It is justified by a large number of simulations that the concept of observability and its computational algorithms can be used as a tool to evaluate the effectiveness of synchrophasor networks. For a 9-bus model with three generators, a single synchrophasor makes the entire system observable at the boundary of stability. Using the data from a single synchrophasor, all angles and angular velocities can be estimated using virtue sensors such as a UKF. The computation shows that observability is not
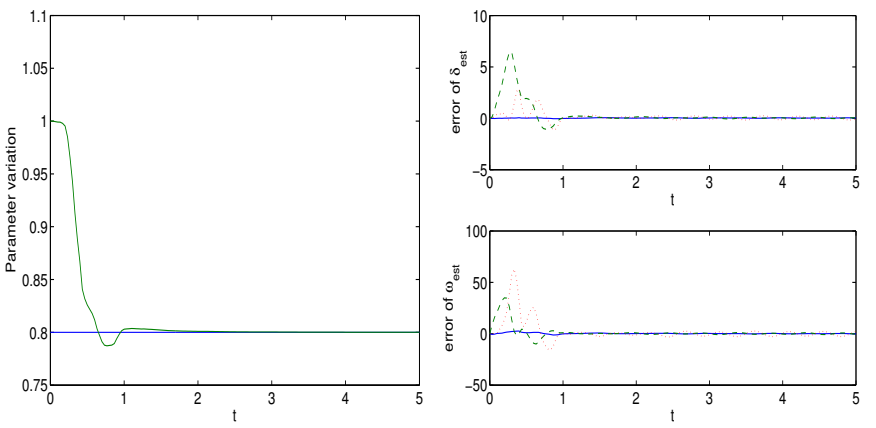

Fig. 8. Adaptive estimation: unknown $Y_{12}$

robust to model uncertainties. However, the unknown parameter variations are observable. It implies that adaptive estimation method should be used to provide reliable state estimates.

Since the 9-bus power system model can be considered as a reduced model of the WECC power system, it indicates that the results and conclusions from the study of the 9bus power system is potentially applicable to a large-scale power grid having multiple control regions interconnected by tie-lines with synchrophasors installed in only some of those regions. For future research, the concept and algorithms will be applied to real system models with hundreds of buses and tens of generators. The work will be focused on the computation of observability and the development of real-time estimation algorithms. The goal is to find the number and the siting of synchrophasors that make the system observable for the detection of instability.

\section{REFERENCES}

W. Kang and L. Xu. Computational Analysis of Control Systems Using Dynamic Optimization. arXiv: 0906.0215v2, 2009.

W. Kang and L. Xu. A Quantitative Measure of Observability and Controllability. Proceedings of IEEE Conference on Decision and Control, Shanghai, China, December, 2009.

W. Kang and L. Xu. Optimal placement of mobile sensors for data assimilations. Tellus A, 64, 17133, 2012.

Q. Gong, W. Kang and I. M. Ross. A Pseudospectral Method for the Optimal Control of Constrained Feedback Linearizable Systems. IEEE Transactions on Automatic Control, Vol. 51, No. 7, pp. 1115-1129, 2006.

S. J. Julier and J. K. Uhlmann. Unscented filtering and nonlinear estimation. Proceedings of the IEEE, Vol. 92, No. 3, pp. 401-422, 2004.

P. M. Anderson and A. A. Fouad. Power Systems Control and Stability, IEEE Press, 1994.

W. Kang and K. Sun. Detection of Instability Using Synchrophasors: A Theoretical Investigation on Observability with Synchrophasor Networks. EPRI Technical Report, No. 3002002061, 2013.

W. Kang. The consistency of partial observability for PDEs. arXiv: $1111.5846 \mathrm{v} 1,2011$.

F. Fahroo and I. M. Ross. Costate Estimation by a Legendre Pseudospectral Method. Proceedings of the AIAA Guidance, Navigation and Control Conference, 10-12 August 1998, Boston, MA. 